\title{
Dimer Radical Cations of Indole and Indole-3-carbinol: Localized and Delocalized Radical Cations of Diindolylmethane
}

\author{
Anna Błoch-Mechkour and Thomas Bally* \\ Department of Chemistry, University of Fribourg, CH-1700, Fribourg, Switzerland \\ Andrzej Marcinek \\ Institute of Applied Radiation Chemistry, Technical University, 90-924 Lodz, Poland
}

\begin{abstract}
Extending our previous study on the title species (J. Phys. Chem. A 2010, 114, 6787), we investigated the dimer cations that are formed on oxidation of the glucobrassin derivatives indole-3-carbinol (I3C) and diindolylmethane (DIM) and of parent indole (I). Radiolysis in ionic liquid and Ar matrices shows that, at sufficiently high concentrations and/

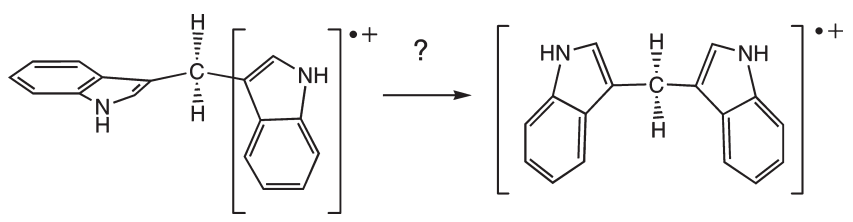
or on annealing the solid glasses, intense intermolecular charge-resonance (CR) absorption bands in the NIR herald the formation of sandwich-type dimer cations. The molecular and electronic structure of these species is modeled by calculations with the doublehybrid B2-PLYP-D density functional method which yields predictions in good accord with experiment. The radical cation of DIM also shows a CR band, but unlike in the case of I and I3C, its occurrence is not dependent on the concentration but instead on the solvent: in ionic liquid the CR band is initially absent and arises only on annealing, whereas in Ar matrices it is present from the outset and undergoes blue shifting and sharpening on annealing. These puzzling findings are rationalized on the basis of B2-PLYP-D calculations which predict that neutral DIM exists in the form of two conformers, present in different relative amounts in the two experiments, which on vertical ionization form distinct radical cations, a nonsymmetric one where the odd electron is largely localized on one of the two indole moieties and one with $C_{2}$ symmetry where charge and spin are completely delocalized over both halves of the molecule, thus giving rise to an intramolecular CR transition. On annealing, the nonsymmetric cation relaxes to a similarly delocalized structure with $C_{s}$ symmetry, thus explaining the observed increase and the shift of the CR band. We believe that DIM ${ }^{\circ+}$ represents the first example of a radical cation which can exist under the same conditions as a localized and a delocalized complex cation.
\end{abstract}

\section{INTRODUCTION}

It is a well-known property of planar $\pi$ systems, such as polyenes and (hetero)aromatic compounds, that upon ionization under suitable conditions they can form $\pi$-complexes $(\mathbf{M})_{2}{ }^{\bullet}$, composed of a radical cation $\mathbf{M}^{\bullet+}$ and its parent, neutral $\mathbf{M}$ in which spin and charge are fully delocalized over both moieties. ${ }^{1}$ Such dimer radical cations are bound by substantial covalent or resonance contributions $\Delta E_{\text {res }}$, arising mainly from the interaction between the HOMO of $\mathbf{M}$ and the SOMO of $\mathbf{M}^{\mathbf{}}$ as depicted in Scheme 1.

Spectroscopically $(\mathbf{M})_{2}{ }^{\bullet+}$ can be identified by having broad, intense, near-infrared bands which are attributed to so-called "charge-resonance" (CR) transitions of energy $E_{\mathrm{CR}}$. ${ }^{2}$ These transitions can be understood on the basis of the generic MO diagram in Scheme 1, which shows how the MOs of $\mathbf{M}$ and $\mathbf{M}^{\bullet+}$ split as the two species interact. The lowest energy electronic excitation in the resulting dimer radical cations is that between the bonding and the antibonding combination of the two occupied frontier MOs. Charge-resonance (CR) transitions are usually quite intense because the oscillator strengths for excitations of that type are high and very broad because the distance between the interacting $\pi$ systems increases strongly on CR
Scheme 1. MO Picture of the Electronic Structure of Dimer Radical Cations $(\mathbf{M})_{2}^{\bullet+}$

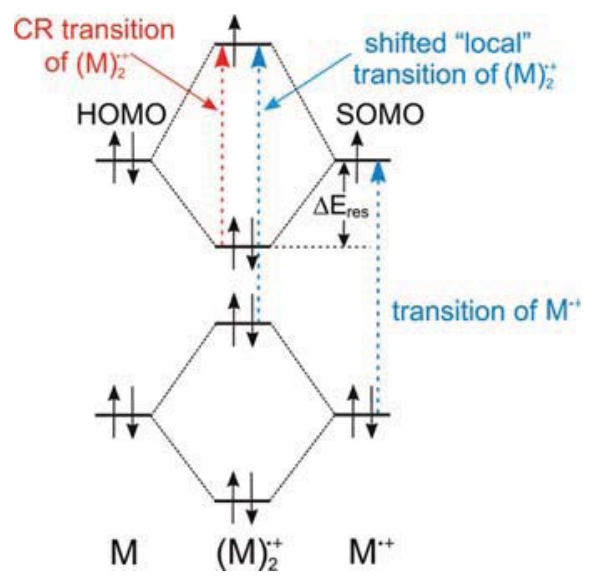


Scheme 2

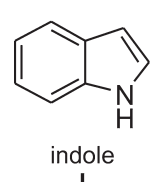

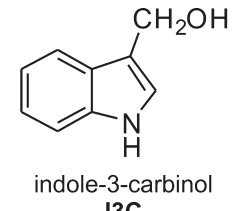

I3C

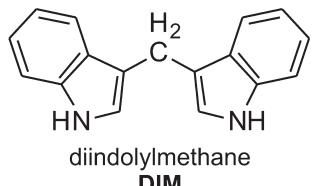

DIM excitation, as in such transitions an electron is promoted from an $\mathrm{MO}$ that is bonding between the two moieties to one that is antibonding. In addition to CR bands, dimer radical cations $(\mathbf{M})_{2}{ }^{\bullet+}$ show "local" transitions which are related to those of the monomer radical ions $\mathbf{M}^{\bullet+}$ but are usually shifted by some amount.

In the course of our work on the oxidation products of two naturally occurring glucobrassicin metabolites, indole-3-carbinol (I3C) and diindolylmethane (DIM), ${ }^{3}$ we noticed that in samples of high concentration (in Ar matrices) and/or upon annealing of matrices containing the radical cations a broad NIR band appears, indicating that these compounds can form dimer radical cations. In the case of DIM the question arises whether that transition arises by intra- or intermolecular complexation.

In this paper we present the absorption spectra of these dimer radical cations, obtained by annealing frozen ionic liquid solutions of parent indole (I, I3C, or DIM) irradiated with electron pulses or $\mathrm{X}$-irradiated Ar matrices (I, DIM). We assign these spectra in terms of possible structures of the complex dimer radical cations (I, I3C), and we discuss in particular the conditions under which DIM forms a radical cation where the charge is localized in one indole moiety or where it is delocalized over both of them. The discussion is based on quantum chemical calculations by methods which are able to account for the mix of covalent and nonbonding interaction that prevail in dimer cations.

\section{EXPERIMENTAL AND COMPUTATIONAL METHODS}

Materials. Indole and its derivatives were obtained from Sigma/ Aldrich and purified by sublimation before use. The ionic liquid, 1-butyl-3-methylimidazolium hexafluorophosphate $\left(\mathrm{BMIM}^{+} \mathrm{PF}_{6}^{-}\right){ }^{4}$ was prepared according to literature procedures.

Matrix Isolation and Spectroscopy. ${ }^{5}$ Ground crystals of indole (I) were placed in a U-tube attached to the inlet system of an ATR closed cycle cryostat and carefully degassed. A 9:1 mixture of argon and nitrogen (where the latter is added to improve the optical quality of matrices) was passed at ca. 0.5 Torr through the U-tube held at $8{ }^{\circ} \mathrm{C}$ and slowly deposited on a CsI window maintained at ca. $20 \mathrm{~K}$. The much less volatile DIM was sublimed from a double-walled glass tube fitted to the vacuum shroud of the cryostat. The opening of the inner tube which faces the cold CsI window is surrounded by four nozzles through which $\mathrm{Ar}$ is directed to the same window to ensure efficient mixing with the substance emanating from the heated tube. To deposit DIM to a similar concentration to that of $\mathbf{I}$ in the matrix, the inner glass tube was resistively heated to $122{ }^{\circ} \mathrm{C}$. Unfortunately, I3C could not be volatilized to a sufficiently high concentration to form dimers (and hence dimer cations after ionization) in the resulting matrices.

To generate radical cations, the $\mathrm{Ar} / \mathrm{N}_{2}$ mixture was doped with about $1 \%$ of $\mathrm{CH}_{2} \mathrm{Cl}_{2}$, which acts as an electron scavenger. The sample was exposed to $90 \mathrm{~min}$ of $\mathrm{X}$-irradiation from a tungsten target bombarded at $40 \mathrm{kV} / 40 \mathrm{~mA}$. The following net processes are thought to occur

$$
\begin{gathered}
\mathrm{Ar}+h v(\mathrm{X}) \rightarrow \mathrm{Ar}^{\bullet+}+\mathrm{e}^{-} \\
\mathrm{CH}_{2} \mathrm{Cl}_{2}+\mathrm{e}^{-} \rightarrow \mathrm{CH}_{2} \mathrm{Cl}^{\bullet}+\mathrm{Cl}^{-} \\
\mathrm{Ar}^{\bullet+}+\mathbf{S} \rightarrow \mathrm{S}^{\bullet+}+\mathrm{Ar}(\mathbf{S}=\mathbf{I} \text { or } \mathbf{D I M})
\end{gathered}
$$

Measurements in Ionic Liquid. Glassy solutions of indole derivatives in a mixture of 1-butyl-3-methylimidazolium hexafluorophosphate and methylene chloride $\left(\mathrm{BMIM}^{+} \mathrm{PF}_{6}{ }^{-}: \mathrm{CH}_{2} \mathrm{Cl}_{2}, 1: 1\right.$ $\mathrm{v} / \mathrm{v}$ ) were prepared by immersing room-temperature solutions into liquid nitrogen in cuvettes with an optical path length of 0.5$3 \mathrm{~mm}$. These cuvettes were then placed in a liquid nitrogen-cooled cryostat (Oxford Instruments), where any temperature between 77 and $300 \mathrm{~K}$ can be maintained by controlled heating. The optical absorption spectra were measured on a Cary 5 (Varian) spectrometer. The samples mounted in the cryostat were irradiated with $4 \mu$ s electron pulses from an ELU-6 linear accelerator. The spectra presented in the figures of this paper are invariably difference spectra (after minus before irradiation).

We have shown previously that ionic liquids, many of which form stable transparent glasses at $77 \mathrm{~K}^{6}$ can be used for generating radical ions by radiolysis. Recently, we found that the glass quality does not change upon mixing $\mathrm{BMIM}^{+} \mathrm{PF}_{6}^{-}$with $\mathrm{CH}_{2} \mathrm{Cl}_{2}$, which has the advantage that it improves the solubility of many precursors and leads to a higher yield of radical cations on radiolysis due to its ability to scavenge electrons by dissociative attachment. ${ }^{4}$

Quantum Chemical Calculations. The geometries of all species were optimized first by the well-established B3LYP/ 6-31G* method by which stationary points were also characterized by frequency calculations. Following that all geometries were reoptimized by the double-hybrid dispersion-corrected B2PLYP-D density functional method, ${ }^{7,8}$ as implemented in the Gaussian 09 suite of programs, ${ }^{9}$ using Dunning's-type correlation-consistent cc-pVDZ basis set. ${ }^{10,11}$ This method is designed to account both for the covalent bonding as well as for mid- and long-range correlation effects which contribute to the binding in molecular complexes such as dimer cations. As analytic second derivatives are not available yet for double-hybrid functional methods, frequency calculations were not possible at the B2-PLYP level. The $\pi$-dimer radical cations of I and I3C were optimized using the Boys-Bernardi counterpoise (CP) method $^{12,13}$ to correct for basis set superposition errors (BSSE). Excited state energies were calculated by density functional-based time-dependent linear response theory ${ }^{14}$ using the TD-B2-PLYP method.

\section{RESULTS AND DISCUSSION}

3.1. Dimer Radical Cations of Indole, $\left(\mathrm{I}_{2}{ }^{\bullet+}\right.$, and Indole-3carbinol, $(\mathrm{I} 3 \mathrm{C})_{2}^{\cdot+}$. Figure 1a shows the absorption spectrum of ionized indole in a $\mathrm{BMIM}^{+} \mathrm{PF}_{6}{ }^{-} / \mathrm{CH}_{2} \mathrm{Cl}_{2}$ matrix. The intense peak at $330 \mathrm{~nm}$ and the double-humped band at 560/605 nm have been previously attributed to the radical cation of indole. ${ }^{3}$ Softening of the irradiated frozen solution by controlled increase of the temperature to $120-140 \mathrm{~K}$ allows for slow diffusion to take place. This leads to the decay of the absorption bands of $\mathbf{I}^{\bullet+}$ (Figure $1 \mathrm{~b}$ and $1 \mathrm{c}$ ), which is accompanied by the buildup of a broad structureless band extending from 600 to $1400 \mathrm{~nm}$ and 


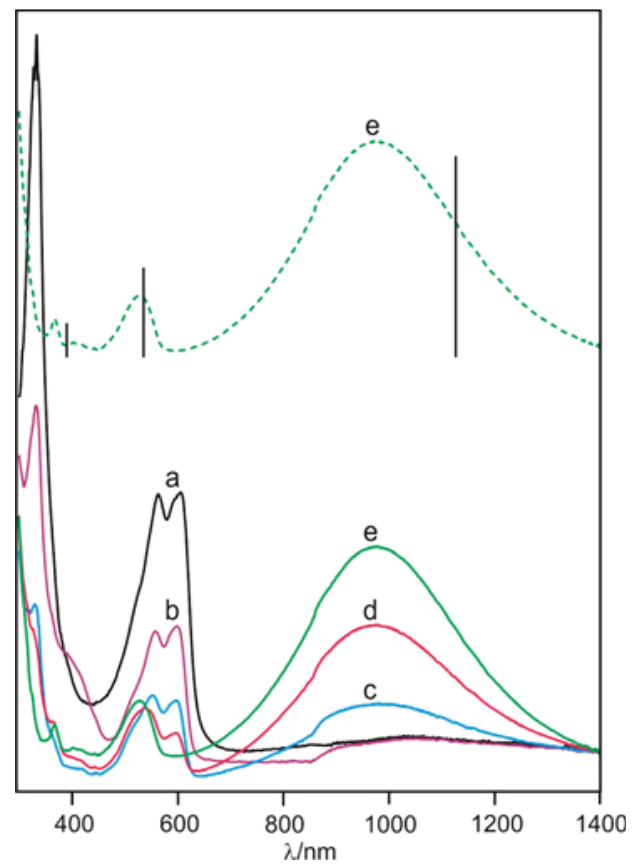

Figure 1. Spectrum obtained (a) on radiolysis of a $0.02 \mathrm{M}$ solution of I in a 1:1 mixture of $\mathrm{BMIM}^{+} \mathrm{PF}_{6}{ }^{-}$and $\mathrm{CH}_{2} \mathrm{Cl}_{2}$ at $77 \mathrm{~K}$ and (b) after annealing at $120 \mathrm{~K}$ for $30 \mathrm{~min}$, (c) $130 \mathrm{~K}$ for $30 \mathrm{~min}$, (d) $135 \mathrm{~K}$ for $15 \mathrm{~min}$, and (e) $135 \mathrm{~K}$ for $55 \mathrm{~min}$; black bars represent results from TDDFT calculations for the most stable dimer radical cation of $\mathbf{I}$ (see text for discussion).

peaking at ca. $980 \mathrm{~nm}$. After the absorptions of $\mathrm{I}^{\bullet+}$ have decayed completely (Figure 1e), a new band at $525 \mathrm{~nm}$ and a small peak at $365 \mathrm{~nm}$ can be discerned. Upon annealing to $>140 \mathrm{~K}$, all three bands decay concomitantly. We attribute the spectrum in Figure le to the dimer radical cation complex formed by a neutral molecule of indole and its radical cation, $(\mathrm{I})_{2}{ }^{\bullet+}$.

For I3C the sequence of events is similar: the absorption spectrum of $\mathrm{I} 3 \mathrm{C}$ in a $\mathrm{BMIM}^{+} \mathrm{PF}_{6}{ }^{-} / \mathrm{CH}_{2} \mathrm{Cl}_{2}$ matrix (Figure 2a) shows also an intense peak at $330 \mathrm{~nm}$ and a wider, doublehumped band peaking at ca. $600 \mathrm{~nm}$ which we assigned to $\mathrm{I} \mathrm{C}^{\circ+3}$. Upon annealing of the glass, first to $160 \mathrm{~K}$ and finally to $165 \mathrm{~K}$, a decay of the absorption bands of $\mathrm{I}^{3} \mathrm{C}^{\circ+}$ is observed (Figure $2 \mathrm{~b}-\mathrm{e}$ ). At the same time a broad NIR band extending from 600 to $1600 \mathrm{~nm}$ and peaking at ca. $1200 \mathrm{~nm}$ grows in. In addition, we can discern a band peaking at $565 \mathrm{~nm}$ which may already be contained in the spectrum obtained after radiolysis or could have arisen on annealing (Figure $2 \mathrm{e}$ ). We attribute the spectrum in Figure $2 \mathrm{e}$ to the I3C dimer radical cation. The concomitant decrease of these bands on annealing above $165 \mathrm{~K}$ indicates that they belong to the same species.

In an effort to shed more light on these processes, we decided to subject $\mathrm{I}$ to ionization in an $\mathrm{Ar}$ matrix at $10 \mathrm{~K}$. If such a matrix containing a high concentration of $\mathrm{I}$ is subjected to $\mathrm{X}$-radiolysis, a broad CR band due to $(\mathrm{I})_{2}{ }^{\bullet+}$ appears in the NIR next to the absorptions of monomeric $\mathbf{I}^{\bullet+}$ (see Figure $3 \mathrm{~b}$ ). Interestingly, this $\mathrm{CR}$ band is broader and red shifted by about $0.23 \mathrm{eV}$ compared to that obtained on annealing an ionic liquid glass (cf. Figure 3c), which may indicate that under these experimental conditions other and perhaps more conformations of $(\mathrm{I})_{2}{ }^{\bullet+}$ are formed. However, annealing of the Ar matrix to $35 \mathrm{~K}$ did not affect the shape and position of this band.

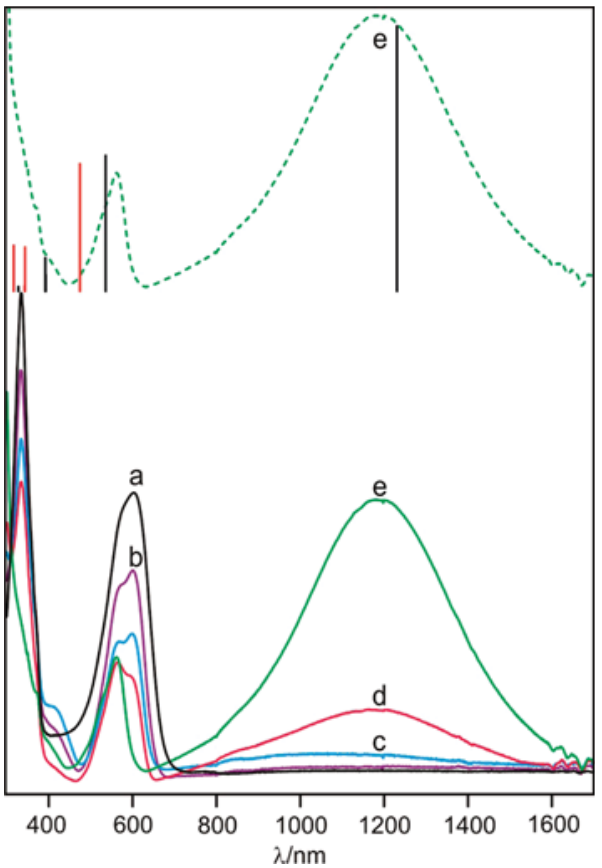

Figure 2. Spectrum obtained (a) on radiolysis of a frozen $0.02 \mathrm{M}$ solution of $\mathrm{I} 3 \mathrm{C}$ in $\mathrm{BMIM}^{+} \mathrm{PF}_{6}{ }^{-} / \mathrm{CH}_{2} \mathrm{Cl}_{2}$ at $77 \mathrm{~K}$ and $(\mathrm{b})$ after annealing at $160 \mathrm{~K}$ for $10 \mathrm{~min},(\mathrm{c}) 160 \mathrm{~K}$ for $30 \mathrm{~min},(\mathrm{~d}) 160 \mathrm{~K}$ for $50 \mathrm{~min}$, and (e) $165 \mathrm{~K}$ for $10 \mathrm{~min}$; black and red bars represent results from TD-DFT calculations for two the most stable dimer radical cation of I3C (see text for discussion).

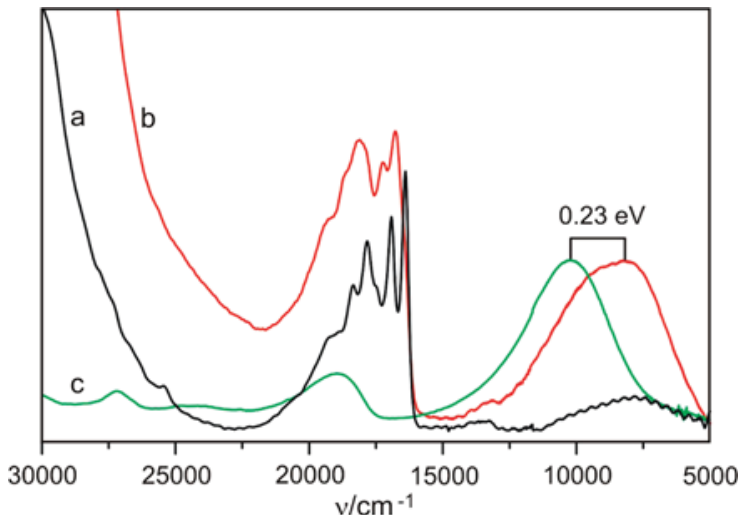

Figure 3. (a) Spectrum of $\mathbf{I}^{+\bullet}$ obtained in Ar matrix (1:1000) at $10 \mathrm{~K}$; (b) the same at concentration of 1:200; (c) spectrum of $\mathrm{I}$ ionized in $\mathrm{BMIM}^{+} \mathrm{PF}_{6}^{-} / \mathrm{CH}_{2} \mathrm{Cl}_{2}$ after annealing at $135 \mathrm{~K}$ for $55 \mathrm{~min}$.

To identify possible structures of the dimer cations, $(\mathbf{I})_{2}{ }^{\bullet+}$ and $(\mathrm{I} 3 \mathrm{C})_{2}{ }^{\bullet+}$, we resorted to quantum chemical calculations. The geometries of different dimer radical cation structures were optimized by the B2-PLYP-D/cc-pVDZ method, accounting for the BSSE correction (cf. Experimental and Computational Methods section). The most stable of these structures $(\mathrm{I})_{2}{ }^{\bullet+}$ and $(\mathrm{I} 3 \mathrm{C})_{2}{ }^{++}$are shown in Tables 1 and 2 , respectively.

First, we note that these dimer cations are bound by up to $1 \mathrm{eV}$, which shows that forces much larger than those which prevail in van der Waals complexes hold these species together. For $(\mathrm{I})_{2}{ }^{\bullet+}$ we found that structure $\mathbf{A}$, where the best overlap between the two indole rings prevails, has the lowest energy. 
Table 1. Relative $\left(E_{\text {rel }}\right)$ and Binding $\left(E_{\text {bind }}\right)$ Energies of Different Structures of $(\mathrm{I})_{2}{ }^{\bullet+}$

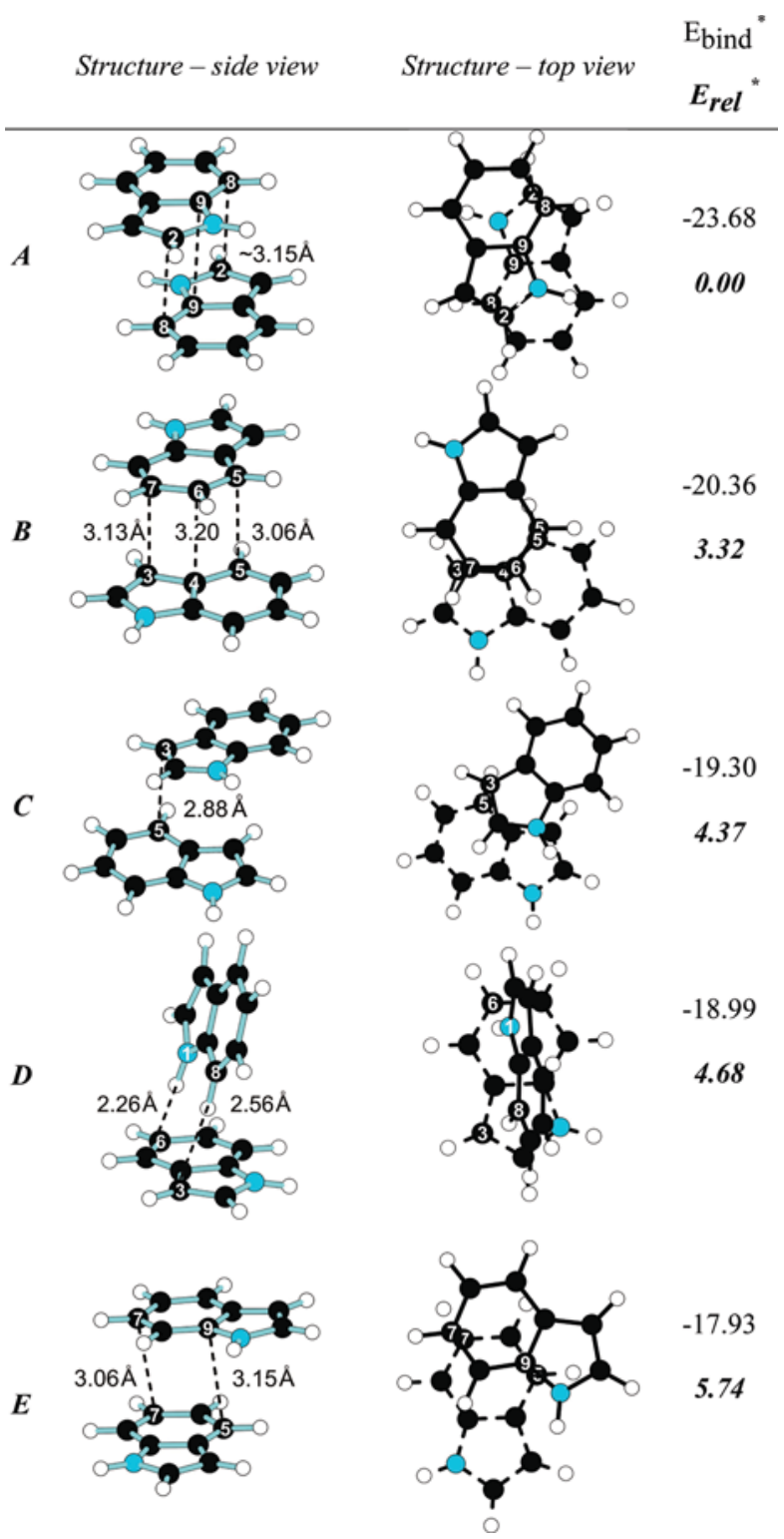

* Relative to the sum of the energy of neutral I $(-363.459928 \mathrm{Ha})$ and $\mathbf{I}^{\bullet+}(-363.187207 \mathrm{Ha})$.

Interestingly, one of the most stable structures (D) is not of the $\pi$-complex type but seems to be bound by $\mathrm{H}$ bonds. We also did TD-DFT calculations for the NIR transitions of all five structures of $(\mathrm{I}) 2^{\bullet+}$ listed in Table 1 , and we found that the electronic transitions predicted for the most stable structure $\mathbf{A}$ are in the best agreement with the experimental spectrum (see black bars in Figure 1; other transitions are listed in the Supporting Information).

The similarity of the spectra in Figures $1 \mathrm{e}$ and $2 \mathrm{e}$ indicates that the structure of $(\mathrm{I} 3 \mathrm{C})_{2}{ }^{++}$may be akin to that of $(\mathrm{I})_{2}{ }^{\bullet+}$, although our calculations (Table 2) predict that a $\mathrm{N}-\mathrm{H} \cdots \mathrm{O}(\mathrm{H})$ bonded structure (B) actually has the lowest energy. However, TD-DFT calculations predict that this structure has only absorptions below $600 \mathrm{~nm}$ (see red bars in Figure 2), whereas the predictions for structure $\mathbf{A}$ of $(\mathbf{I} 3 \mathrm{C})_{2}{ }^{\cdot+}$ (black bars in Figure 2), which shows
Table 2. Relative $\left(E_{\text {rel }}\right)$ and Binding $\left(E_{\text {bind }}\right)$ Energies of Different Structures of $(\mathrm{I} 3 \mathrm{C})_{2}{ }^{\bullet+}$

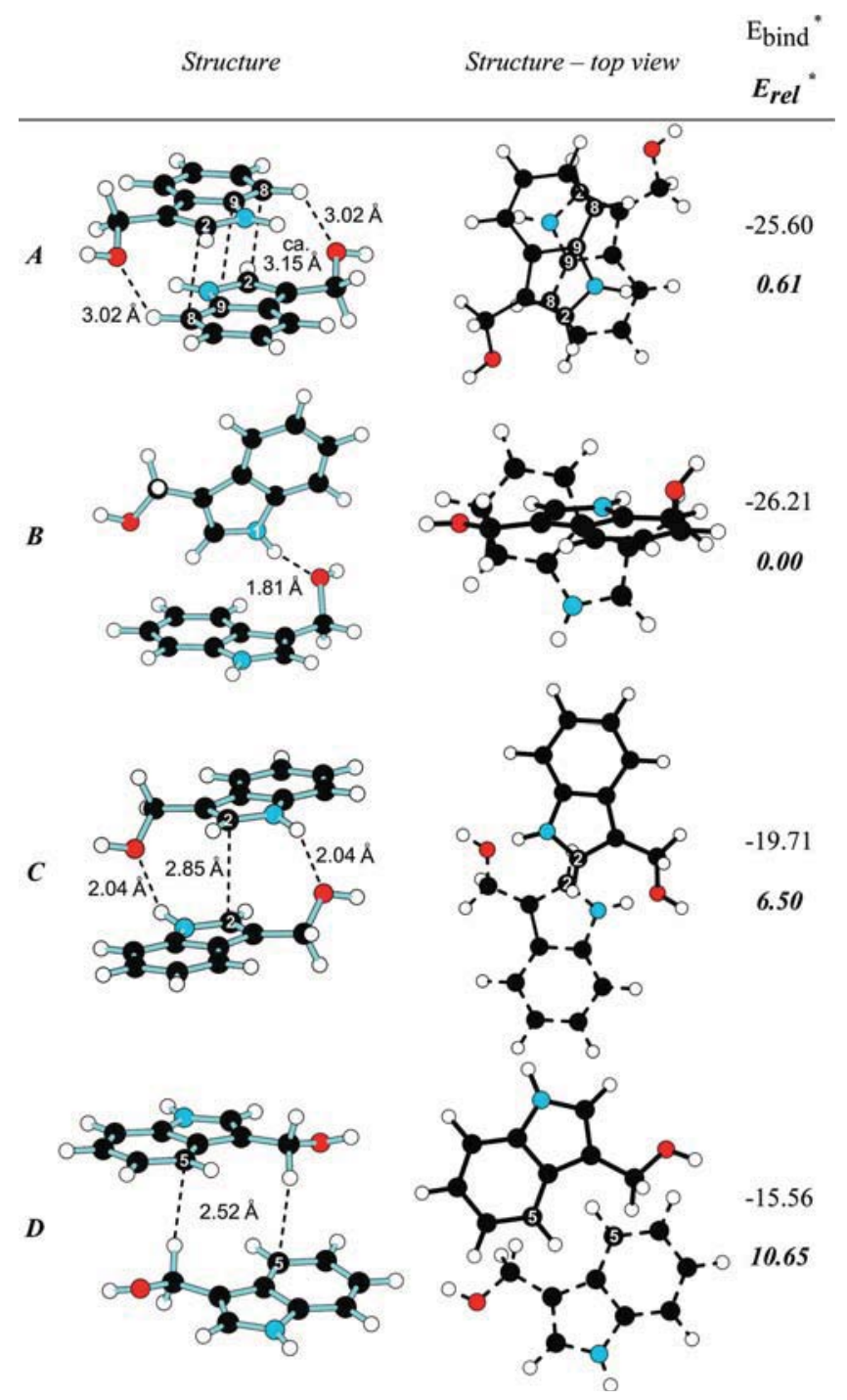

* Relative to the sum of energy of neutral I3C $(-477.887461 \mathrm{Ha})$ and $\mathbf{I}^{3} \mathbf{C}^{\bullet+}(-477.625022 \mathrm{Ha})$.

great similarity to structure $\mathbf{A}$ of $(\mathbf{I})_{2}{ }^{\bullet+}$, show good agreement with the experimentally observed CR band. Nevertheless, it cannot be excluded that upon annealing of ionic liquid glass we also obtain some of structure $\mathbf{B}$ of $(\mathbf{I 3 C})_{2}{ }^{\bullet+}$.

The electronic structure of conformers $\mathbf{A}$ of $(\mathbf{I})_{2}{ }^{\bullet+}$ and $(\mathrm{I} 3 \mathrm{C})_{2}{ }^{+}$is presented in Table 3 in terms of excitations involving the molecular orbitals shown in Figure 4. As expected for both $(\mathrm{I})_{2}{ }^{\bullet+}$ and $(\mathrm{I} 3 \mathrm{C})_{2}{ }^{\bullet+}$ the NIR band corresponds to the first excited state, which is a charge-resonance transition, attained essentially by promotion of an electron from the $\mathrm{HOMO}-1$ to the singly occupied MO (SOMO). These orbitals are composed of the bonding and antibonding combinations of the singly occupied MOs of monomeric radical cations of $\mathbf{I}$ for $(\mathbf{I})_{2}{ }^{\cdot+}$ and $\mathbf{I} 3 \mathrm{C}$ for $(\mathrm{I} 3 \mathrm{C})_{2}{ }^{\bullet+}$, respectively. The band in the visible region, which corresponds to the fourth excited state, is due a socalled "local" transition related to those of the monomer radical cations of $\mathbf{I}^{\bullet+}$ and $\mathbf{I} 3 \mathbf{C}^{\bullet+}$, respectively. Allowed transitions below $400 \mathrm{~nm}$ cannot be associated with leading excitations. 
Table 3. Excited States of Structures $A$ of $(\mathrm{I})_{2}{ }^{\bullet+}$ (bold) and $(\mathrm{I} 3 \mathrm{C})_{2}{ }^{\bullet+}$ (italic) Calculated by TD-B2PLYPD/cc-pVDZ

\begin{tabular}{|c|c|c|c|c|c|}
\hline \multirow{3}{*}{$\begin{array}{l}\text { excited state } \\
\text { first }\end{array}$} & \multicolumn{2}{|c|}{ energy } & \multirow[b]{2}{*}{ oscillator strength } & \multirow{2}{*}{\multicolumn{2}{|c|}{ main excitation }} \\
\hline & \multirow{2}{*}{$\begin{array}{c}\mathrm{eV} \\
1.10\end{array}$} & \multirow{2}{*}{$\begin{array}{c}\mathrm{nm} \\
\mathbf{1 1 2 6}\end{array}$} & & & \\
\hline & & & 0.1595 & $61 \beta \rightarrow 62 \beta$ & 0.98948 \\
\hline & 1.01 & 1229 & 0.1542 & $77 \beta \rightarrow 78 \beta$ & 0.99046 \\
\hline second & 1.27 & 978 & 0 & $60 \beta \rightarrow 62 \beta$ & 0.99408 \\
\hline & 1.39 & 891 & 0 & $76 \beta \rightarrow 78 \beta$ & 0.99288 \\
\hline third & 1.70 & 730 & 0.001 & $59 \beta \rightarrow 62 \beta$ & 0.97374 \\
\hline & 1.80 & 688 & 0.0013 & $75 \beta \rightarrow 78 \beta$ & 0.94981 \\
\hline fourth & 2.31 & 536 & 0.0703 & $58 \beta \rightarrow 62 \beta$ & 0.96791 \\
\hline & 2.30 & 538 & 0.0768 & $74 \beta \rightarrow 78 \beta$ & 0.94052 \\
\hline fifth & 3.17 & 391 & 0.0258 & highly mixed & \\
\hline & 3.13 & 396 & 0.0093 & highly mixed & \\
\hline sixth & 3.19 & 388 & 0.0011 & highly mixed & \\
\hline & 3.14 & 395 & 0.019 & highly mixed & \\
\hline seventh & 3.47 & 357 & $\mathbf{0}$ & $57 \beta \rightarrow 62 \beta$ & 0.92114 \\
\hline & 3.43 & 362 & 0 & $73 \beta \rightarrow 78 \beta$ & 0.82567 \\
\hline eighth & 3.71 & 334 & 0.004 & highly mixed & \\
\hline & 3.74 & 331 & 0.0026 & highly mixed & \\
\hline
\end{tabular}

3.2. Radical Cations Derived from DIM. In order to dissolve DIM to a concentration of $0.01 \mathrm{M}$ in $\mathrm{BMIM}^{+} \mathrm{PF}_{6}{ }^{-}$the ionic liquid has to be mixed again with an equal part of $\mathrm{CH}_{2} \mathrm{Cl}_{2}$. The spectrum obtained after radiolysis of this solution at $77 \mathrm{~K}$ (Figure 5a) shows a very similar double-humped band around $600 \mathrm{~nm}$, as observed with I under the same conditions (cf. Figure 1a). This band can readily and unequivocally be assigned to a DIM radical cation $\left(\mathbf{D I M}^{\boldsymbol{O}^{+}}\right.$) where spin and charge are localized in one of the indole moieties, while the other indole is just a "spectator" substituent.

Upon annealing the sample, this spectrum changes in a way which indicates formation of several secondary products, some of which we previously identified, ${ }^{3}$ i.e., the red dye urorosein (the band peaking at $490 \mathrm{~nm}$ in the spectrum in Figure 5e) and the C-centered radical of DIM (assigned to a small peak at $420 \mathrm{~nm}$ ). A third species, which we did not discuss in the previous paper, has a broad, structureless band in the NIR region characteristic for charge-resonance (CR) transitions of dimer radical cations (cf. section 3.1), and very similar to the band observed for the dimer cation of $\mathbf{I} 3 \mathrm{C}$ in $\mathrm{BMIM}^{+} \mathrm{PF}_{6}{ }^{-}$(cf. Figure 2).

This would seem to indicate that a similar species is formed, i.e., that the indole moiety of an ionized DIM molecule which carries the spin (and formally the charge) forms a complex with one of the indole moieties of a nearby neutral DIM, with the remaining two indole moieties serving as uninvolved substituents. However, we believe that this is not the case, because the exact same NIR band appears on annealing independently of the concentration of DIM (down to $5 \mathrm{mM}$, below which no bands can be detected), whereas $20 \mathrm{mM}$ solutions are needed to obtain clearly discernible dimer cations on annealing of solutions of ionized I and I3C. Also, one would expect the NIR band to be present from the outset in concentrated solutions, if it is due to intermolecular complex formation. Thus, we looked for alternative explanations.

The occurrence of a CR band in a radical cation containing two identical chromophores which may interact through space or via orbitals of the bridging moiety (a $\mathrm{CH}_{2}$ group in the case of DIM)

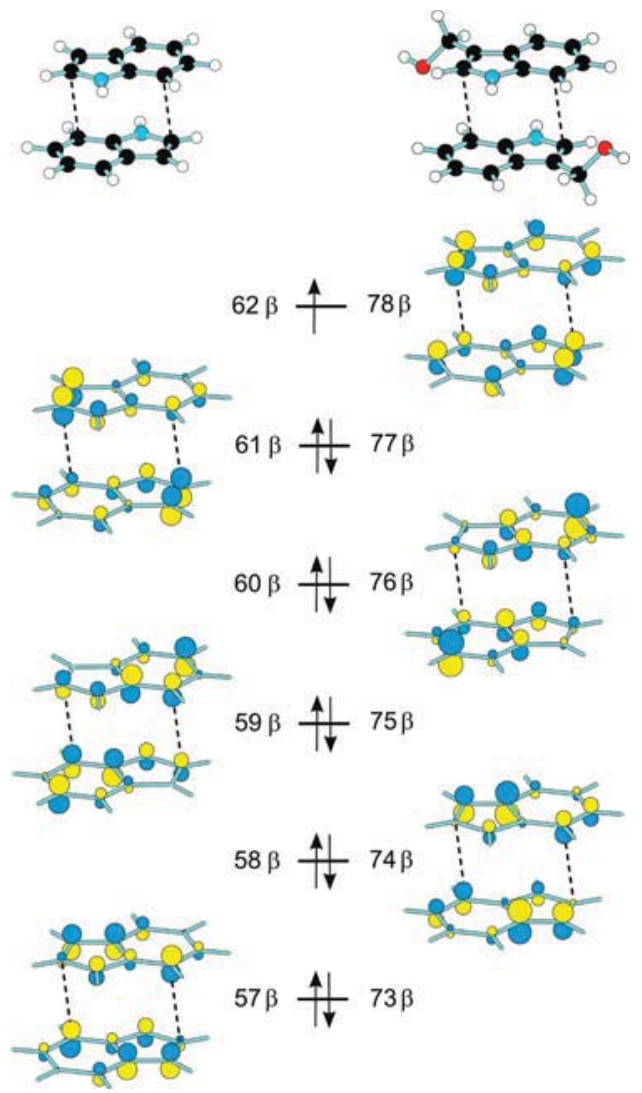

Figure 4. Kohn-Sham molecular orbitals of $(\mathbf{I})_{2}{ }^{\bullet+}$ (numbers to the left) and $(\mathbf{I} 3 \mathrm{C})_{2}{ }^{++}$(numbers to the right) involved in the electronic transitions of these species (cf. Table 3 ).

and thus form an intramolecular dimer radical cation is not unprecedented (a simple example is the radical cation of norbornadiene ${ }^{15}$ where two olefins interact intramoleculary). As the $\mathrm{CH}_{2}$ link is too short to allow for effective through-space overlap of the $\pi$ systems of the two indole moieties, this effect will lead to a smaller splitting of their frontier MOs than in $(\mathrm{I})_{2}{ }^{\bullet+}$. It is therefore to be expected that the CR band in $\operatorname{DIM}^{\bullet+}$ will be at lower energy, which is indeed what is observed. However, what is intriguing about the observations reported in Figure 5 is that a localized radical cation (which has no CR transitions) seems to be converted into a delocalized form (which has a strong CR transition) on annealing, a rather exceptional phenomenon that calls for an explanation.

As in the case of $\mathbf{I}$ we decided to ionize DIM also in an $\mathrm{Ar}$ matrix at $10 \mathrm{~K}$, which resulted in the spectrum shown in Figure 6a. In contrast to the experiment in the ionic liquid glass, this spectrum comes in two distinct parts, one of which is almost identical to that of $\mathrm{I}^{\bullet+}$ in $\mathrm{Ar}$ (cf. Figure 3a), whereas the other consists of a very broad, CR-type NIR band. It is important to note that, judging from the intensities of comparable IR bands, the concentration of DIM in the Ar matrix was similar to that of I before ionization, i.e., it was such that intermolecular dimer cations are not preformed in the matrix (which is the case at much higher concentrations, cf. Figure $3 b$ ). We therefore believe that the NIR band of ionized DIM in Ar is not due to intermolecular dimer cations, which are not formed under such conditions.

However, this CR band peaks at significantly lower energy $\left(\lambda_{\max } \approx\right.$ $1700 \mathrm{~nm})$ than the one in ionic liquid glass $\left(\lambda_{\max } \approx 1300 \mathrm{~nm}\right)$ 


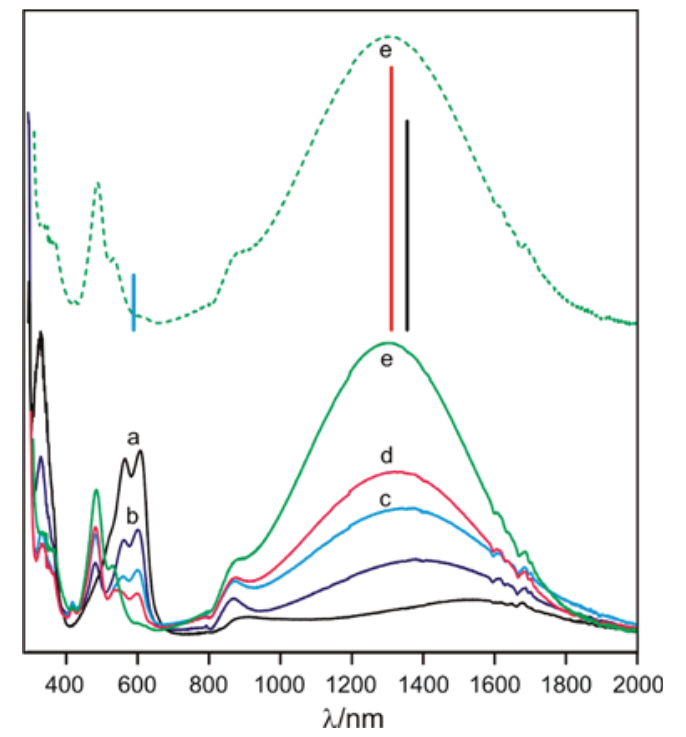

Figure 5. Spectrum obtained (a) on radiolysis of a $0.01 \mathrm{M}$ solution of DIM in a 1:1 mixture of $\mathrm{BMIM}^{+} \mathrm{PF}_{6}{ }^{-}$and $\mathrm{CH}_{2} \mathrm{Cl}_{2}$ at $77 \mathrm{~K}$ and (b) after annealing at $135 \mathrm{~K}$ for $10 \mathrm{~min}$, (c) $135 \mathrm{~K}$ for $30 \mathrm{~min}$, (d) $135 \mathrm{~K}$ for $40 \mathrm{~min}$, and (e) $145 \mathrm{~K}$ for $10 \mathrm{~min}$; black, red, and blue bars represent results from TD-B2PLYP calculations on DIM radical cations (see text for discussion).

and extends much further toward the IR range. Upon annealing above $20 \mathrm{~K}$, we first observe a concomitant decay of the sharp band system at $600 \mathrm{~nm}$ and a low-energy tail (above $2000 \mathrm{~nm}$ ) while the CR transition sharpens and shifts to higher energies. Subsequent annealing up to $25 \mathrm{~K}$ results in further narrowing and blue shifting of the NIR band with no further decay of the $600 \mathrm{~nm}$ band system.

As there can be no doubt that the $600 \mathrm{~nm}$ band system must be assigned, as in the above case of the experiments in ionic liquid, to a localized DIM radical cation and that the intense NIR band belongs most probably to a delocalized form of the same cation (because formation of intermolecular dimer cations can be practically excluded at the concentrations which prevailed in the Ar matrix), we are led to conclude that the two forms actually coexist in Ar matrices. It thus seems that the energetic advantages of localizing or delocalizing spin and charge, respectively, strike a very delicate balance in the case of $\mathrm{DIM}^{+}$, a balance which can tip one way or the other depending on subtle differences in geometry or the environment.

In the ionic liquid the balance seems to be initially in favor of the localized form, perhaps because this enjoys better solvation in this polar solvent. However, the conformational freedom gained by $\mathbf{D I M}^{\bullet+}$ on annealing appears to allow this compound to attain geometries where the delocalized form is more stable, as indicated by the appearance of the NIR band, again at lower energy than in Ar because in this solid conformational restrictions prevent complete relaxation even upon annealing.

\section{THEORETICAL STUDIES ON LOCALIZED AND DELO- CALIZED DIM ${ }^{+}$}

Unfortunately, the balance between localized and delocalized forms of radical cations such as $\operatorname{DIM}^{\circ}+$ cannot be assessed unambiguously by current quantum chemical methods: as delocalization of the spin is "driven" by the energy gained upon

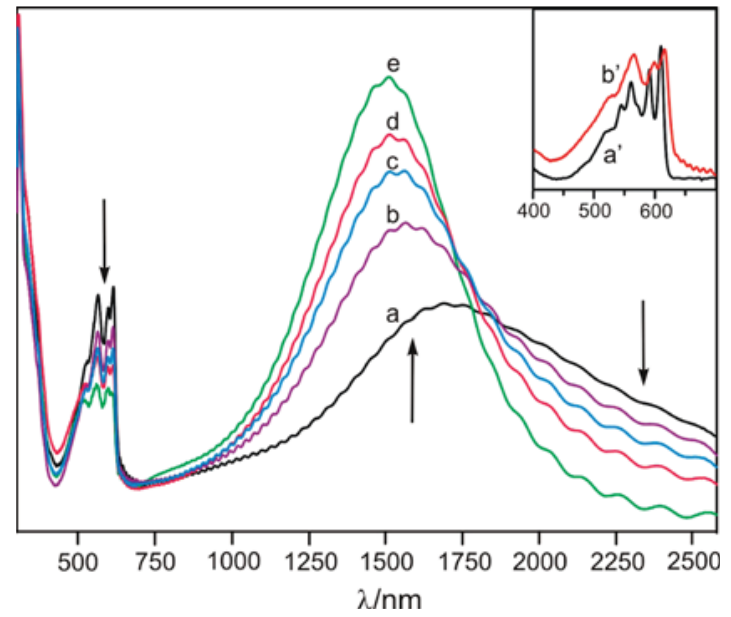

Figure 6. (a) Spectrum of DIM ionized in an Ar matrix at $10 \mathrm{~K}$ and (b) after annealing at $20 \mathrm{~K}$ for $15 \mathrm{~min}$, (c) $23 \mathrm{~K}$ for $15 \mathrm{~min}$, (d) $25 \mathrm{~K}$ for $15 \mathrm{~min}$, and (e) $25 \mathrm{~K}$ for $15 \mathrm{~min}$. The wiggles in the NIR are due to interferences. (Inset) Spectrum of DIM $\left(a^{\prime}\right)$ and I $\left(b^{\prime}\right)$ ionized in $\operatorname{Ar}$ matrix at $10 \mathrm{~K}$.

correlating the movements of the electrons, Hartree-Fock (or CASSCF) theory invariably predicts localized radical cations to be more stable than delocalized ones. Accounting for these correlation effects by means of many-body perturbation theory (MBPT), such as in the popular MP2 (or CASPT2) method, fails because the change on going from a fully localized to a fully delocalized wave function is too large to be handled as a perturbation. On the other hand, density functional theory artifactually overestimates the stability of delocalized radical cations, because delocalization minimizes the spurious self-interaction of the odd electron which arises by the incomplete cancellation of the classical Coulomb and the exchange energy for a single electron for all currently known exchange functionals. ${ }^{16}$

In addition to these molecule-inherent effects, solvation will favor localization of the partial charge in radical cations (which to some degree accompanies spin localization) and thus create a bias in favor of the localized form of ionized DIM. In view of all this we cannot hope to make a reliable theoretical prediction of the relative stability of the localized and delocalized forms of ionized DIM, even in the gas phase, let alone in a condensed medium.

What we can, however, do is model the delocalized form of ionized DIM by DFT. Thus, we explored the conformational space of DIM and its radical cation by B2-PLYP-D/cc-pVDZ. In Figure 7 the conformational minima of DIM and of its radical cation are presented. The neutral has two equilibrium conformations of very similar energy, one with no symmetry, where the planes of the two indole moieties are nearly perpendicular, $\operatorname{DIM}\left(C_{1}\right)$, and one of nearly $C_{2}$ symmetry, $\operatorname{DIM}\left(C_{2}\right)$.

On removing an electron from the HOMO, the vertically formed radical cation of $\operatorname{DIM}\left(C_{1}\right)$ relaxes to a structure which has nearly $C_{s}$ symmetry while that obtained by ionization of $\operatorname{DIM}\left(C_{2}\right)$ keeps its 2-fold axis of symmetry and relaxes to the structure denoted $C_{2}$-a in Figure 7. However, this structure is protected by a barrier of only $2.85 \mathrm{kcal} / \mathrm{mol}$ from further relaxation to structure $C_{2}$-b, which lies ca. $1.28 \mathrm{kcal} / \mathrm{mol}$ lower in energy. All the structures can be characterized by the two $\mathrm{C}-\mathrm{C}-\mathrm{C}-\mathrm{C}$ dihedral angles $d_{1}$ and $d_{2}$ around the bonds linking the two indole moieties to the bridging $\mathrm{CH}_{2}$ group, which are given in Figure 7. 


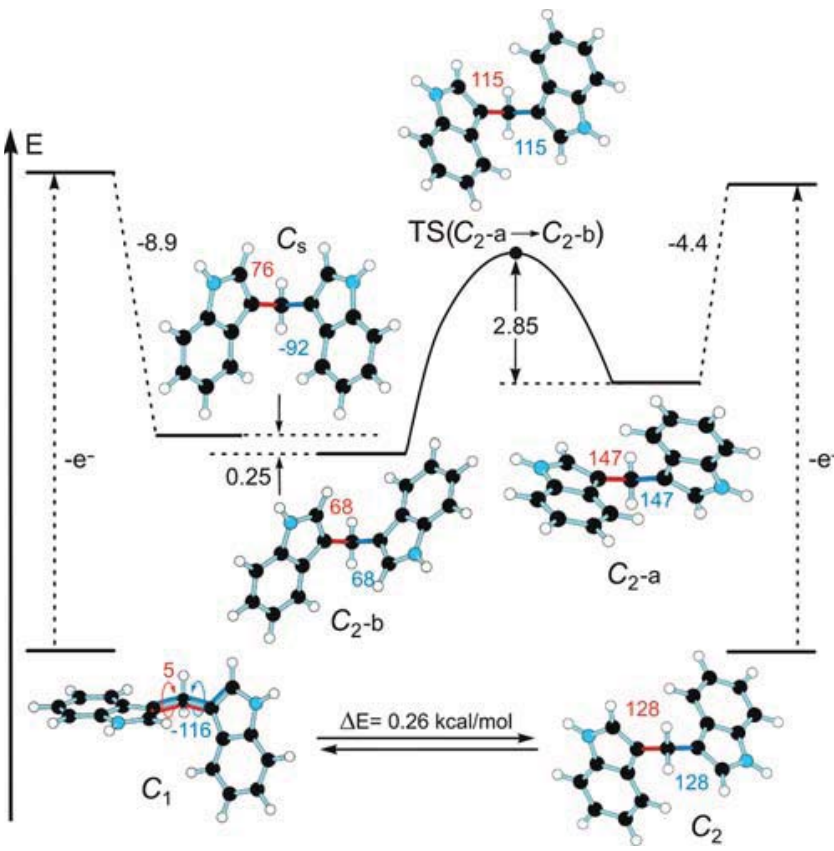

Figure 7. Equilibrium structures of DIM and DIM $^{\bullet+}$ (relative energies in $\mathrm{kcal} / \mathrm{mol}$ ).

Table 4. Main Electronic Transitions Predicted by TDB2PLYP/cc-pVDZ for DIM ${ }^{\bullet+}$

\begin{tabular}{ccccccc} 
& \multicolumn{2}{c}{ energy } & & & \\
conformer & $\mathrm{eV}$ & $\mathrm{nm}$ & oscillator strength & \multicolumn{2}{c}{ main excitation } \\
\cline { 2 - 3 }$_{s}$ & 0.91 & 1357 & 0.1976 & $64 \beta \rightarrow 65 \beta$ & 0.98111 \\
$C_{2}$-a & 0.39 & 3191 & 0.0964 & $64 \beta \rightarrow 65 \beta$ & 0.99676 \\
& 2.09 & 593 & 0.0517 & $61 \beta \rightarrow 65 \beta$ & 0.97324 \\
$C_{2}$-b & 0.94 & 1313 & 0.2485 & $64 \beta \rightarrow 65 \beta$ & 0.97681 \\
\hline
\end{tabular}

In the $C_{2}$ and the $C_{s}$ conformations of $\mathbf{D I M}^{\bullet+}$ charge and spin are of course fully delocalized over both indole moieties. For both the $C_{s}$ and the $C_{2}$-b conformations of the radical cation, TDB2PLYP-D calculations predict an intense CR-type transition around $1300 \mathrm{~nm}$ (cf. Table 4), arising from HOMO-1 to SOMO $(64 \rightarrow 65)$ excitation (see MOs in Figure 8$)$. These predictions are in very good agreement with the observed NIR band obtained upon annealing in ionic liquid glass (cf. Figure 5).

In contrast, for the initially formed $C_{2}$-a conformer a much weaker CR transition is predicted at ca. $3000 \mathrm{~nm}$. The reduced splitting of the two highest MOs is due to an inversion of the "natural sequence" of orbitals (the bonding combination of indole HOMOs lies above the antibonding one, see Figure 8) as a consequence of strong through-bond interaction involving pseudo- $\pi$ MOs of the bridging $\mathrm{CH}_{2}$ group.

In order to localize transition states between all three conformers of $\mathrm{DIM}^{\bullet+}$ we generated a 3D potential energy surface by scanning the two $\mathrm{C}-\mathrm{C}-\mathrm{C}-\mathrm{C}$ dihedral angles $d_{1}$ and $d_{2}$ (see Figure $\mathrm{S} 2$ in the Supporting Information). This surface indicates where the transition states are expected to lie, and we had indeed no problem locating that for the interconversion of the two $C_{2}$ conformers, shown in Figure 7 as $\operatorname{TS}\left(C_{2}-\mathrm{a} \rightarrow C_{2}-\mathrm{b}\right)$. In the search for the transition states linking the $C_{s}$ to either of the two $C_{2}$ conformers we ran, however, into a problem in that the ground

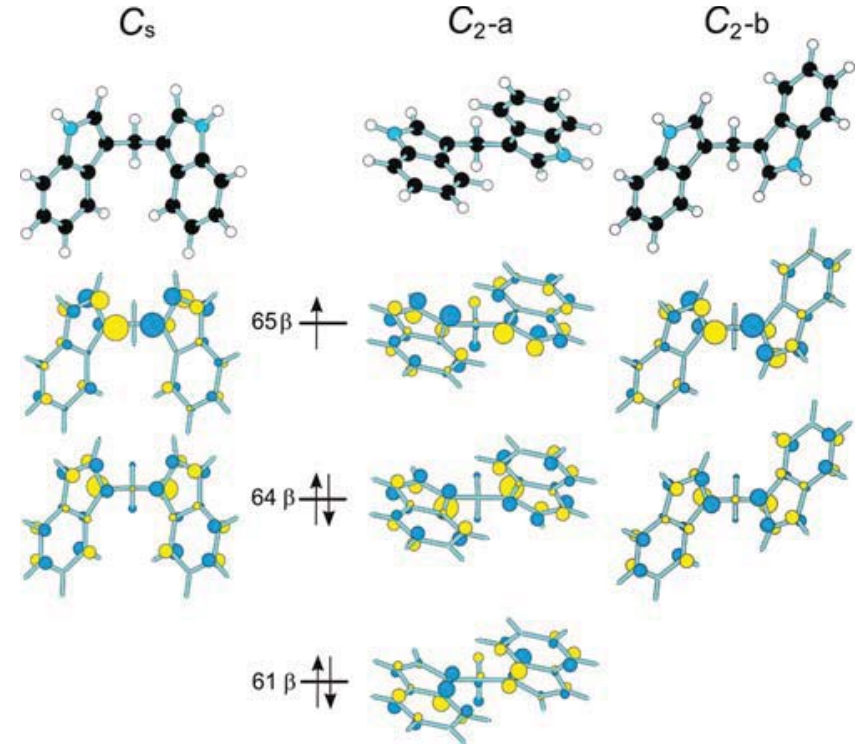

Figure 8. MOs of $\mathrm{DIM}^{\bullet+}$ involved in the electronic transitions of its three conformers (cf. Table 4).

states of reactant conformers connect adiabatically to the excited states of product conformers and vice versa.

This is illustrated in Figure 9, which shows that in both ground states the $\mathrm{SOMO}$ is antibonding across the $\mathrm{CH}_{2}$ group but as one twists the right indole moiety the $\mathrm{SOMO}$ becomes bonding, i.e., one ends up in an excited state of the product radical cation. As geometries along the reaction coordinate have no symmetry, this "orbital following" can be avoided without breaking any symmetry. However, in order to pass from a reactant to a product conformer ground state surface, the coefficients on one of the indole moieties must go to zero, so they can re-emerge with the opposite sign on the product side. Physically, this corresponds to a complete localization of the spin in one-half of the molecule at or near the transition state.

However, such a transition, from a species with delocalized spin to one where the spin is localized in one-half of the molecule, cannot be achieved by DFT or MBPT methods, ${ }^{17}$ so this transition state cannot be located using currently available methodology. On the basis of the numbers in Figure 9 (which come from TD-DFT calculations $)^{18}$ we predict that the energy of TS $\left(C_{S} \rightarrow C_{2}\right.$-b) should be less than $10 \mathrm{kcal} / \mathrm{mol}$ above the conformers of delocalized DIM $^{\bullet+}$. Thus, it cannot be excluded that the $C_{\mathrm{s}}$ and $C_{2}$-b conformers interconvert to some extent before the incipient radical cations are thermalized.

Even if we accept the above assignments, several questions remain: (a) why does the broad CR band in the NIR only appear after annealing in the ionic liquid glass, whereas it is present from the outset in the Ar matrix spectrum; (b) what is the nature of the low-energy tail in the NIR band, which disappears upon slightly raising the temperature, in $\mathrm{Ar}$, and (c) what is the origin of the ca. $0.14 \mathrm{eV}$ shift of the CR transition of $\mathrm{DIM}^{\bullet+}$ between annealed ionic liquid and $\mathrm{Ar}$ (cf. Figure 10).

In order to answer these questions, we have to address the issue of what happens on vertical ionization of the most stable conformer of DIM, $C_{1}$ in Figure 7. Surely, at this geometry the spin will not be distributed evenly between the two indole moieties and may in fact remain localized on one of the two until the geometry has a chance to relax and at least approach that of the species labeled $C_{s}$ in Figure 7 . If we assume that this 


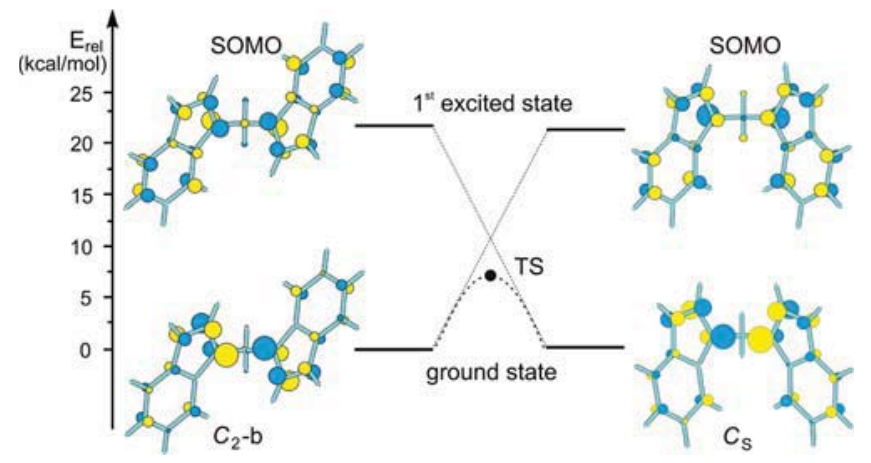

Figure 9. Apparent state crossing between the $C_{2}$-b and the $C_{s}$ conformers of DIM $^{\bullet+}$; numbers are based on (TD)B2PLYPD calculations (the two ground states are within $0.25 \mathrm{kcal} / \mathrm{mol}$ ).

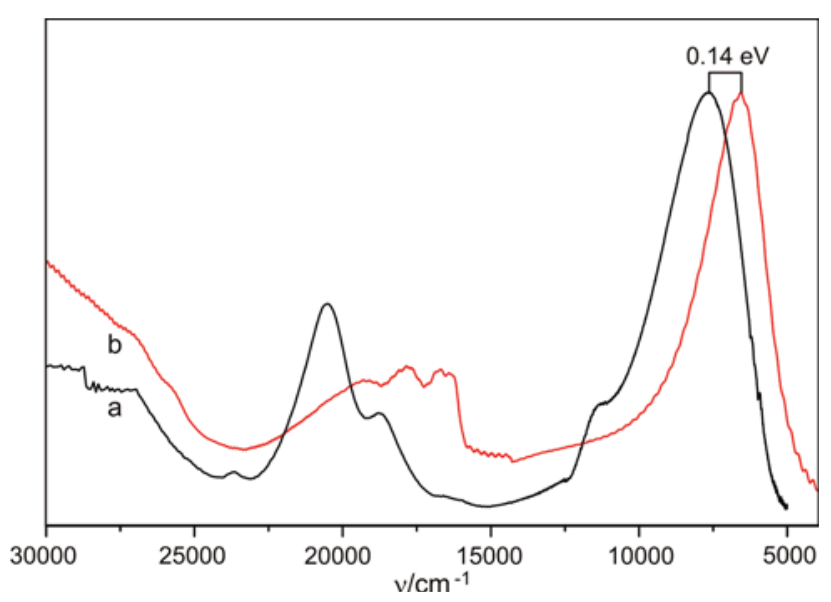

Figure 10. (a) Spectrum obtained on radiolysis of a $0.01 \mathrm{M}$ solution of DIM in a 1:1 mixture of $\mathrm{BMIM}^{+} \mathrm{PF}_{6}{ }^{-}$and $\mathrm{CH}_{2} \mathrm{Cl}_{2}$ after annealing at $145 \mathrm{~K}$ for $10 \mathrm{~min}$; (b) spectrum of DIM ionized in Ar matrix after annealing at $25 \mathrm{~K}$ for $15 \mathrm{~min}$. Note that the peak at $19000 \mathrm{~cm}^{-1}$ is due to the urorosein cation. ${ }^{3}$

relaxation, which involves a $70^{\circ}$ rotation of one of the indole moieties, is inhibited in solid matrices and can only take place upon their gradual annealing, this may explain why the initial spectrum obtained upon ionization of DIM in an ionic liquid glass and a part of the initial spectrum obtained in Ar has the characteristics of a radical cation where only one of the indole moieties is ionized while the other is acting as a "spectator" substituent. ${ }^{19}$ Upon annealing, that spectrum would be gradually replaced by one of a species with delocalized spin and charge, i.e., the CR band that is characteristic for such species.

So why is this CR band present from the outset in Ar but arises only after annealing the ionic liquid? An answer to this question might be found in the thermochemistry of the equilibrium between the two conformers of neutral DIM $\left(\Delta H_{\text {calcd }}=+\right.$ $0.26 \mathrm{kcal} / \mathrm{mol}, \Delta S_{\text {calcd }}=-1.91 \mathrm{cal} \mathrm{K}^{-1} \mathrm{~mol}^{-1}$ for $\left.C_{1} \rightarrow C_{2}\right)$.

In the ionic liquid experiment, the equilibrium will be rapidly established, at least during the initial phases of the cooling process. At $77 \mathrm{~K}$, where $\Delta G_{\text {calc }, 77 \mathrm{~K}}=0.41$ and $K=0.07$ (assuming that $\Delta H$ and $\Delta S$ do not change between 298 and $77 \mathrm{~K}$ ), $93 \%$ of the DIM is present as conformer $C_{1}$, i.e., the localized radical cation is expected to predominate largely before its relaxation (and concomitant delocalization) to the $C_{\mathrm{s}}$ structure. In contrast, in order

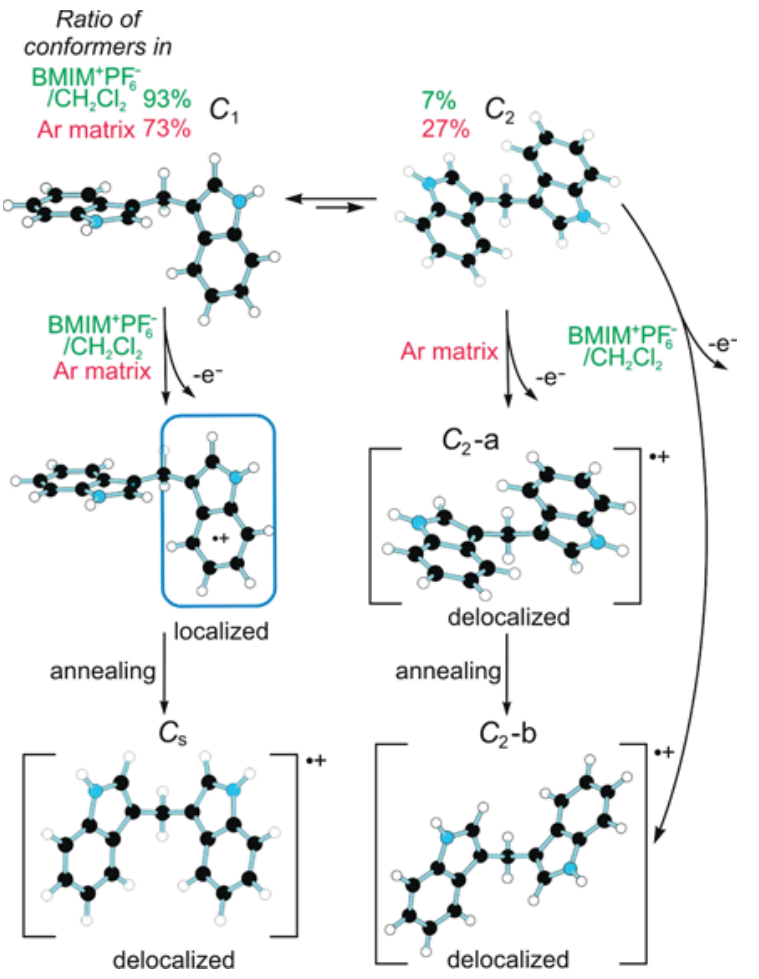

Figure 11. Processes that occur in Ar matrix and ionic liquid glass upon radiolysis and further annealing.

to get DIM into the gas phase for Ar matrix experiments, the material must be heated to ca. $120^{\circ}$ where $\Delta G_{\text {calcd,395K }}=$ $1.01 \mathrm{kcal} / \mathrm{mol}, K=0.21$, i.e., $27 \%$ of the DIM is present as conformer $\mathrm{C}_{2}$. As vertical ionization of this conformer leads already to a symmetric (i.e., delocalized) radical cation, this may explain the presence of a CR band in the Ar matrix experiments right after ionization of DIM (if $\Delta H$ is larger than the calculated value, the effect will be more pronounced).

The presence of the thermally labile low-energy tail of the NIR transition in Ar may be rationalized by the initial presence after ionization of some $\mathrm{DIM}^{\bullet+}$ in the metastable conformation $C_{2}$-a which has a CR transition at much lower energy than the other conformations (cf. Table 4). Slight annealing will permit relaxation of this to the more stable conformation $C_{2}$-b, which is formed directly on annealing the ionic liquid glass to $160 \mathrm{~K}$. Alternatively, one may argue that the transition between the localized $C_{1}$ and the delocalized $C_{\mathrm{s}}$ conformation is not a stepwise one but that intermediary, partially localized conformations may be attained and initially trapped in $\mathrm{Ar}$ (but not in the ionic liquid glass). These conformations are expected to have $\mathrm{CR} / \mathrm{CT}$ transitions at lower energy than the symmetric, fully relaxed ones. Our experimental data do not allow us to distinguish between these two possible interpretations.

Finally, we propose that the shift of the CR band between the ionic liquid and Ar may be due to one or both of two effects: (a) the presence of substantial amounts of conformer $C_{2}$-b of $\operatorname{DIM}^{\bullet+}$, which may have a lower energy CR transition than conformer $C_{s}$, in the Ar matrix experiments (see above) and/or (b) enhanced stabilization of the CR excited state of DIM $^{\bullet+}$ by the highly polarizable Ar environment.

Figure 11 sums up the processes that we believe to occur upon ionization of DIM in an ionic liquid glass and in Ar, respectively, based on the experimental spectra and DFT calculations. 


\section{CONCLUSIONS}

We investigated the radical cations obtained on pulse radiolysis of frozen ionic liquid solutions of parent indole (I), indole3-carbinol (I3C), and diindolylmethane (DIM) or on X-irradiation of Ar matrices of I and DIM, respectively. On annealing or at high concentration, I and I3C form dimer radical cations which distinguish themselves by intense charge-resonance (CR) bands in the NIR range which were assigned on the basis of quantum chemical calculations. The behavior of DIM on ionization is more complicated: initially it forms a radical cation where the spin is localized in one of the two indole moieties that are connected by a $\mathrm{CH}_{2}$ group. On annealing, this species is gradually transformed into one where spin and charge are symmetrically delocalized over both indole moieties and which shows a CR band similar to that of the dimer cations of I and I3C. Due to the increased presence of a higher lying symmetric conformer of neutral DIM prior to ionization in the Ar matrix experiments, a CR band is already seen right after ionization. Thus, DIM is one of the rare cases where a localized and a delocalized radical cation may coexist. The subtleties of the DIM radical cation are discussed on the basis of quantum chemical calculations of the potential energy surfaces and the excited states.

\section{ASSOCIATED CONTENT}

S Supporting Information. Pdf file containing (a) the complete citation of the Gaussian program, (b) a table and a figure showing the excited states of conformer $\boldsymbol{B}$ of $(\mathbf{I} \mathbf{C} \mathbf{C}) 2^{\bullet+},(\mathrm{c})$ a table with the equilibrium conformations of DIM and its radical cation, (d) the B3LYP/6-31G* potential energy surface generated by scanning the two dihedral angles $d_{1}$ and $d_{2}$ in DIM $^{\bullet+}$; separate text file containing the geometries and energies of all stationary points mentioned in the paper and detailed results of excited state calculations.

\section{AUTHOR INFORMATION}

\section{Corresponding Author}

*E-mail: Thomas.Bally@unifr.ch.

\section{ACKNOWLEDGMENT}

This work is part of project no. 200020-121747 of the Swiss National Science Foundation. A.M. acknowledges support from the Polish Ministry of Science and Higher Education (no. N205 003 32/0258).

\section{REFERENCES}

(1) Badger, B.; Brocklehurst, B. Trans. Faraday Soc. 1969, 65, 2576

(2) Badger, B.; Brocklehurst, B. Trans. Faraday Soc. 1970, 66, 2939.

(3) Błoch-Mechkour, A.; Bally, T.; Sikora, A.; Michalski, R.; Marcinek, A.; Gebicki, J. J. Phys. Chem. A 2010, 114, 6787.

(4) Czerwinska, M.; Sikora, A.; Szajerski, P.; Zielonka, J.; Adamus, J.; Marcinek, A.; Bednarek, P.; Bally, T. J. Org. Chem. 2000, 71, 5312.

(5) Bally, T. In Reactive Intermediate Chemistry; Moss, R. A., Platz, M. S., Jones, M., Eds.; Wiley \& Sons: New York, 2004.

(6) Marcinek, A.; Zielonka, J.; Gebicki, J.; Gordon, C. M.; Dunkin, I. R. J. Phys. Chem. A 2001, 105, 9305.

(7) Grimme, S. J. Chem. Phys. 2006, 124, 034108

(8) Schwabe, T.; Grimme, S. Phys. Chem. Chem. Phys. 2007, 9, 3397.
(9) Frisch, M. J.; Trucks, W.; Schlegel, H. B.; Scuseria, G. E.; Robb, M. A.; Cheeseman, J. R.; Montgomery, J. J. A.; Vreven, T.; Kudin, K. N. Gaussian 09, Rev. A02; Gaussian, Inc.: Wallingford, CT, 2009.

(10) Dunning, T. H., Jr. J. Chem. Phys. 1989, 90, 1007.

(11) Kendall, R. A.; Dunning, T. H., Jr.; Harrison, R. J. J. Chem. Phys. 1992, 96, 6796

(12) Boys, S. F.; Bernardi, F. Mol. Phys. 1970, 19, 553.

(13) Simon, S.; Duran, M.; Dannenberg, J. J. J. Chem. Phys. 1996, 105,11024 .

(14) Casida, M. E. In Recent Advances in Density Functional Methods, Part I; Chong, D. P., Ed.; World Scientific Publ. Co.: Singapore, 1995.

(15) Nelsen, S. F.; Trieber, D. A.; Wolff, J. J.; Powell, D. R.; RogersCrowley, S. J. Am. Chem. Soc. 1997, 119, 6873.

(16) Lundberg, M.; Siegbahn, P. E. J. Chem. Phys. 2005, 122, 224103.

(17) Müller, B.; Bally, T.; Gerson, F.; de Meijere, A.; von Seebach, M. J. Am. Chem. Soc. 2003, 125, 13776.

(18) Calculations indicate that the rotational barrier around the indole $-\mathrm{CH}_{2}$ bond in $\mathrm{DIM}^{\bullet+}$ and hence the driving force for relaxation of the $\mathrm{C}_{1}$ structure-once the bond lengths and angles have adopted the optimal values-is quite small. Unfortunately, it is difficult to arrive at reasonable estimates for this quantity by calculations because the transition from a localized to a delocalized radical cation that accompanies this relaxation cannot be modelled reliably by any method that can be applied to a molecule the size of DIM.

(19) In the parlance of mixed valence compounds, species such as the localized DIM radical cation would be called a Robin-Day class I and the delocalized one a class III system. In spite of the fact that electronic excitation of such delocalized systems involves no net charge transfer whatsoever, the $\mathrm{CR}$ transitions in such systems are called "intervalence charge-transfer" (IVCT) absorptions which lends to some confusion. 